\title{
Congenital agenesis of inferior vena cava as a rare cause of deep vein thrombosis: Case report and review of the literature
}

\author{
Haritha Ackula, Kailash Mosalpuria, Pavankumar Tandra* \\ Hematology \& Oncology Division, Lied Transplant Center, University of Nebraska Medical Center, Omaha, Nebraska, USA
}

Received: June 10, 2016

DOI: $10.5430 /$ crim.v3n3p74
Accepted: June 30, $2016 \quad$ Online Published: July 17, 2016

URL: http://dx.doi.org/10.5430/crim.v3n3p74

\begin{abstract}
Inferior vena cava agenesis (IVCA) is an uncommon cause of deep vein thrombosis (DVT). We report the case of a 26-year-old Caucasian female with unilateral iliofemoral DVT. On further evaluation, CT abdomen revealed diminutive inferior vena cava consistent with IVCA. We have used systemic anticoagulation and catheter mediated thrombolysis with success in this case. Physicians should suspect IVCA in patients with extensive DVT especially in younger individuals.
\end{abstract}

Key Words: Deep vein thrombosis, Inferior vena cava agenesis, Catheter directed thrombolysis

\section{INTRODUCTION}

Inferior vena cava agenesis (IVCA) is a rare congenital anomaly and an uncommon cause of deep vein thrombosis (DVT) with an estimated prevalence of $0.0005 \%$ to $1 \%$ in general population. ${ }^{[1]}$ IVCA is usually reported as an incidental finding and accounts for approximately $5 \%$ of cases of unprovoked DVT in individuals under 30 years of age. ${ }^{[2]} \mathrm{We}$ report a case of IVCA presenting with unilateral extensive iliofemoral DVT. The present case illustrates the diagnosis and management of this condition and emphasizes the need to consider IVC anomalies in the differential diagnosis of DVT especially in a young patient.

\section{Case presentation}

A 26-year-old female was admitted to the hospital with a sudden onset of low back pain and left lower extremity swelling of less than 24 hrs duration. The swelling was associated with pain, numbness and discoloration. She was on intermittent oral hormonal contraceptive pill (OCP) for 6 years prior to presentation. She denied any trauma or recent prolonged immobilization. She was a non-smoker with no personal or family history of thrombophilia or thromboembolic events. On admission, her vitals were pulse rate $90 / \mathrm{min}$, blood pressure $121 / 72 \mathrm{mmHg}$, respiration rate $14 / \mathrm{min}$, temperature $36.9^{\circ} \mathrm{C}$. Physical examination revealed diffuse swelling and mottling in her left lower limb extending from upper thigh to the foot. There was mild tenderness and warmth in the affected limb compared to the normal limb. No injuries/varicosities/neurovascular deficits were noted. Her biochemical and hematological investigations were normal except for mild normocytic anemia with hemoglobin 10.6 g/dl, MCV 87.4 fL, Hematocrit 32.5\%. Duplex ultrasound of the left lower extremity showed DVT involving the left common femoral, femoral, popliteal and peroneal veins. A

\footnotetext{
*Correspondence: Pavankumar Tandra, MD; Email: pav.tandra@unmc.edu; Address: Hematology \& Oncology Division, Lied Transplant Center, University of Nebraska Medical Center, Omaha, Nebraska, USA.
} 
contrast CT scan of the pelvis was done to look for the proximal extension of the clot. It revealed thrombosis of the left common iliac, left external and internal iliac veins with a diminutive IVC and dilated gonadal veins and lumbar collaterals. This raised concern for a more proximal IVC obstruc- tion. A congenital absence of IVC with multiple collaterals distal to the liver with prominent azygous and hemiazygous circulation was confirmed on contrast-enhanced CT scan of the abdomen (see Figure 1).
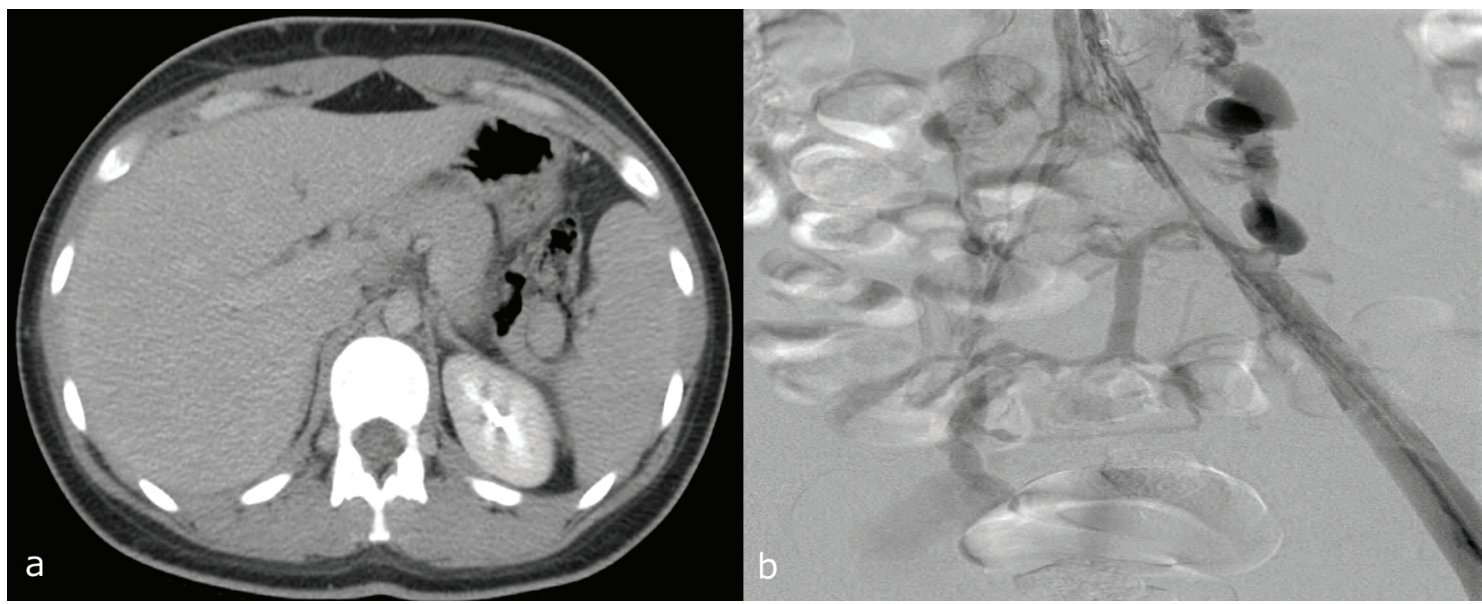

Figure 1. a. Congenitally absent IVC. Note the absence of IVC on the right side; b. Digital subtraction imaging shows thrombosed left iliac vein which does not communicate with the gonadal veins and lower extremity blood flow appears to continue through the azygos, hemizygous and multiple collaterals

Treatment with therapeutic low molecular weight heparin was started promptly on admission. Given her extensive clot burden, a catheter-directed thrombolysis was planned. Patient received continuous thrombolysis with tissue plasminogen activator (tPA) and heparin via the Craig $\mathrm{McNa}-$ mara infusion catheter and a 6 French sheath, respectively. A follow-up venogram demonstrated complete resolution of iliofemoral venous thrombosis with markedly improved flow through the left leg and through the collaterals. No procedural complications were noted and the patient was discharged in a stable condition. She was recommended 6 months of anticoagulation with rivaroxaban, discontinuation of the OCP with the use of two forms of non-pharmacological contraception. Her follow up hospital visit after 2 weeks of discharge showed complete resolution of symptoms with no significant concerns. The patient was receptive to regular follow-ups.

\section{Discussion}

The incidence of venous thrombosis is 1 in 1,000 individuals per year in developed countries. ${ }^{[3]}$ Virchow's triad describes three main causes of thrombosis hypercoagulability, endothelial injury, and venous stasis. ${ }^{[3]}$ The congenital risk factors for thrombosis include deficiencies of protein $\mathrm{C}$, protein S, antithrombin III and gene mutations of prothrombin and factor $\mathrm{V}$ Leiden. The acquired risk factors are surgery, prolonged immobilization, trauma, pregnancy, puerperium, OCP, malignancy and lupus anticoagulant. ${ }^{[3]}$

Published by Sciedu Press
The dynamic interplay of these risk factors induce a state of hypercoagulability and venous stasis, thereby resulting in thrombosis. ${ }^{[3-5]}$ Recently IVC anomalies have been identified as a possible congenital risk factor for DVT. ${ }^{[2,4-7]}$ The estimated prevalence of IVC anomalies is $0.07 \%-8.7 \%$ in the general population. ${ }^{[6]}$ Inferior vena cava develops between $6^{\text {th }}$ to $8^{\text {th }}$ week of embryogenesis from a composite structure formed by sequential appearance, anastomosis and regression of three pairs of primitive veins the posterior cardinal, the subcardinal and the supracardinal veins. ${ }^{[8]}$ Any aberrations in this process result in IVC anomalies which include double IVC, hypoplastic segments of IVC, agenesis of IVC among others. ${ }^{[8]}$ However, there is a school of thought which diverges from this notion and considers chronic occlusion as a consequence of perinatal thrombosis to be the cause of IVC agenesis. ${ }^{[8]}$ Generally, individuals with IVCA develop extensive collateral circulation as a means to compensate for the anomaly. The inadequate blood return through these collaterals results in increased venous blood pressure, which subsequently predisposes to venous thrombosis. ${ }^{[1,4-6]}$

IVCA should be suspected in any young individual presenting with extensive venous thrombosis ${ }^{[1,2,4-7]}$. Chee et al. noted in their case series that up to $5 \%$ to $6.7 \%$ of young adults (aged between 20-40 years) presenting with DVT had an IVC anomaly, which was much higher than the expected $0.5 \% .^{[4]}$ Ruggeri et al. diagnosed four cases of IVCA in patients under 30 years with idiopathic DVT over a 5-year 
period and estimated that IVCA is present in about $5 \%$ of cases of DVT in young adults. ${ }^{[2]}$ Garcia-Fuster et al. in their prospective study of 116 patients under 50 years reported that IVC anomalies were present in $16.2 \%$ of the patients with iliac vein thrombosis and cava malformation represented a risk factor in $5.1 \%$ of the population studied. ${ }^{[7]}$ It is believed that IVC anomalies have been underdiagnosed and may be much more common than anticipated. ${ }^{[2,4]}$ This may be due to the fact that venous ultrasound, most widely used modality for diagnosing DVT is insufficient to detect these anomalies $^{[1,2,5]}$ Contrast enhanced CT scan or MRI studies should be employed in suspected patients. ${ }^{[1,2,5]}$ This is especially effective in the cases involving thrombosis of proximal veins and collaterals masquerading as a malignant mass posing a diagnostic challenge. ${ }^{[5]}$ Venogram is reserved for venous mapping during thrombolysis and for checking post procedural efficacy. ${ }^{[9]}$ Hypercoagulability studies are also recommended; ${ }^{[3-5]}$ however, its relevance is uncertain as it did not modify the patient management in majority of the cases. ${ }^{[1]}$

Since IVCA associated DVT is a rare condition, no specific therapeutic approaches have been developed. The treatment strategy is individualized. Goal of the therapy is to prevent the progression and recurrence of thrombosis. Long-term anticoagulation, elastic stockings and avoidance of risk factors like prolonged immobilization and OCPs have been shown to be of benefit. ${ }^{[1,6]}$ Development of thrombosis in this case is possibly unprovoked since the patient had tolerated intermittent OCP over 6 years without any occurrence of DVT. Six months of systemic anticoagulation was recommended following the discontinuation of the OC pill. In extensive iliofemoral DVT, thrombus removal plus optimal anticoagulation has shown significant improvement in the outcome when compared to anticoagulation alone. ${ }^{[10]}$ Catheter directed intra thrombus delivery of tPA accelerates thrombolysis by preventing neutralization of plasminogen activators by the plasminogen activator inhibitor and also protects the active enzyme plasmin from circulating anti-plasmins. ${ }^{[10]}$ This reduces the overall dose and duration of therapy thereby increasing the efficacy of clot lysis and decreasing the complications when compared to systemic thrombolysis. Catheter directed thrombolysis was also shown to effectively mitigate the clot burden by promoting complete clot resolution, sig- nificant thrombus free survival and reduced post-thrombotic morbidity. ${ }^{[10]}$ Patient underwent catheter-mediated thrombolysis in conjunction with systemic anticoagulation with excellent results (see Figure 2). Literature review of similar cases disclosed successful use of thrombolysis coupled with anticoagulation. ${ }^{[9,11-14]}$ Further studies are warranted to reinforce the role of thrombus removal and its long-term benefit in the treatment of iliofemoral DVT.

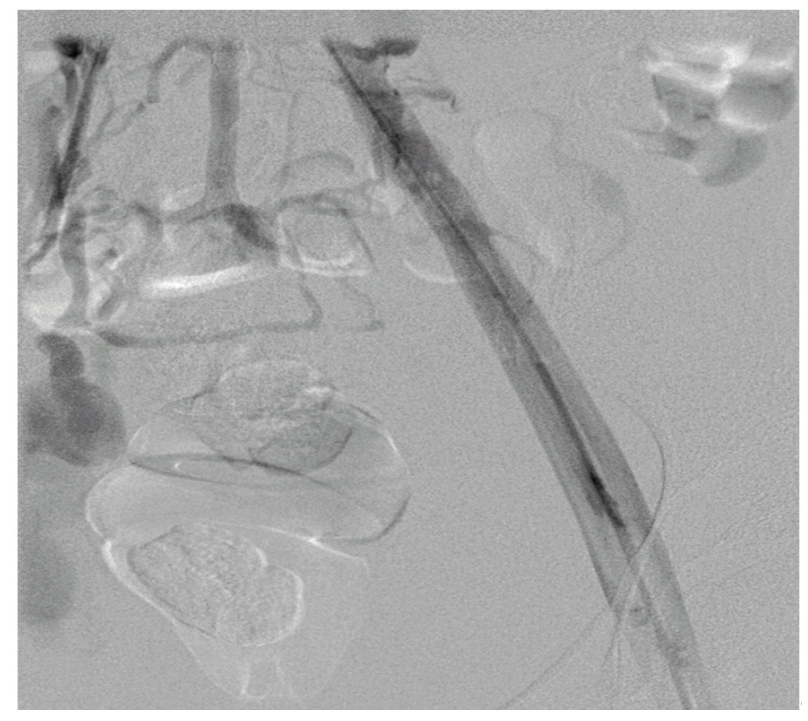

Figure 2. Venogram performed through the left popliteal vein sheath and through the infusion catheter demonstrated complete resolution of iliofemoral venous thrombus with marked improvement in flow through the left leg and through the azygos and pelvic collaterals

\section{Conclusion}

It is reasonable to consider IVCA as a differential diagnosis in young patients presenting with extensive DVT with no typical risk factors. Contrast enhanced CT scan or MRI studies should be considered in diagnosing IVC anomalies. Thrombus removal with optimal anticoagulation has shown to improve the overall outcome in cases of widespread DVT. Avoidance of thrombosis risk factors and use of elastic stockings should be strongly recommended in all DVT patients.

\section{CONFlicts OF INTEREST Disclosure}

The authors have declared no conflicts of interest.

\section{REFERENCES}

[1] Lambert M, Marboeuf P, Midulla M, et al. Inferior vena cava agenesis and deep vein thrombosis: 10 patients and review of the literature. Vascular Medicine. 2010; 15: 451-459. PMid:21183652
http://dx.doi.org/10.1177/1358863X10391355

[2] Ruggeri M, Tosetto A, Castaman G, et al. Congenital absence of the inferior vena cava: a rare risk factor for idiopathic deep-vein thrombosis. The Lancet. 2001; 357(9254): 441. http://dx. doi.o 
rg/10.1016/S0140-6736(00)04010-1

[3] Rosendaal FR. Venous thrombosis: A multicausal disease. The Lancet. 1999; 353(9159): 1167-1173. http://dx.doi.org/10. 1016/S0140-6736 (98) 10266-0

[4] Chee YL, Dominic J, Watson CG, et al. Inferior vena cava malformation as a risk factor for deep vein thrombosis in the young. British Journal of Haematology. 2001; 114: 878-880. PMid:11564079 http://dx . doi.org/10.1046/j.1365-2141.2001.03025.x

[5] Gayer G, Luboshitz J, Hertz M, et al. Congenital anomalies of inferior vena cava revealed on CT in patients with deep vein thrombosis. American Journal of Roentgenology. 2003; 180(3): 729732. PMid:12591684 http://dx.doi.org/10.2214/ajr.180.3 .1800729

[6] Obernosterer A, Aschauer M, Schnedl W, et al. Anomalies of Inferior vena cava in patients with venous thrombosis. Annals of Internal Medicine. 2002; 136(1): 37-41. PMid:11777362 http://dx.doi . org/10.7326/0003-4819-136-1-200201010-00009

[7] Garcia-Fuster MJ, Forner MJ, Flor-Lorente B, et al. Inferior vena cava malformations and deep vein thrombosis. Rev Esp Cardiol. 2006; 59(2): 171-175. PMid:16540040

[8] Bass JE, Redwine MD, Kramer LA, et al. Spectrum of congenital anomalies of the inferior vena cava: Cross-sectional imaging findings. Radiographics. 2000; 20: 639-652. PMid:10835118 http: //dx.doi.org/10.1148/radiographics.20.3.g00ma09639

[9] Singh K, Poliquin J, Syversten G, et al. A rare cause of venous thrombosis: Congenital absence (agenesis) of the inferior vena cava. Int $\mathrm{J}$ Angiol. 2010; 19(3): 110-112. http://dx.doi.org/10.1055/s $-0031-1278377$

[10] Comerota AJ, Gravett MH. Iliofemoral venous thrombosis. J Vasc Surg. 2007; 46: 1065-1076. PMid:17980295 http://dx.doi.org /10.1016/j.jvs.2007.06.021

[11] Fearon WF, Semba CP. Iliofemoral venous thrombosis treated by Catheter-directed thrombolysis, Angioplasty and Endoluminal stenting. West J Med. 1998; 168: 227-279.

[12] Koppisetty S, Smith AG, Dhillon RK. Incidental finding of inferior vena cava atresia presenting with deep vein thrombosis following physical exertion. Case Reports in Emergency Medicine. 2015. Article ID 146304, 4 pages.

[13] Haddad RA, Saadaldin M, Kumar B, et al. Deep vein Thrombosis provoked by Inferior vena cava agenesis. Case reports in Vascular Medicine. 2015. Article ID 651436.

[14] Man L, Hendricks N, Mailand H. IVC agenesis: a rare cause of deep vein thrombosis. Journal of Thrombosis and Thrombolysis. 2016; 41(3): 541-543. PMid:26507132 http://dx.doi.org/10.1007 /s11239-015-1289-0 